\title{
VALIDATION OF IMMUNOPHENOTYPING BY FLOW CYTOMETRY IN THE INVESTIGATION OF DIAGNOSTIC AND PROGNOSTIC MARKERS FOR BREAST CANCER
}

\author{
Daniella Serafin Couto Vieira', Sandro Wopereis, Laura Otto Walter ${ }^{1}$, Maria Claudia Santos da Silva² \\ ${ }^{1}$ Hospital Universitário Professor Polydoro Ernani de São Thiago - Florianópolis (SC), Brazil. \\ 2Departamento de Analises Clinicas, Universidade Federal de Santa Catarina - Florianópolis (SC), Brazil.
}

Introduction: Given its high prevalence, breast cancer has a great financial impact on health systems. Currently, the diagnosis is made by morphological analysis and immunohistochemistry (IHC). However, this methodology has some limitations. Therefore, new methods should be developed to assist those in use, based on the fast and safe detection of tumor cells. Objective: To validate immunophenotyping by flow cytometry (FC) in the investigation of diagnostic and prognostic markers for breast cancer and study lymphocyte subtypes infiltrating the tumor and their relationship with tumor development. Method: A total of 52 samples of breast tumors were sectioned, macerated in phosphate-buffered saline, stained with antibodies against estradiol receptors (ER), progesterone receptors (PR), HER2, Ki67, CD3, CD4, CD8, and CD45, and analyzed by FC. All results were compared with IHC (standard method) as to sensitivity, specificity, positive predictive value (PPV), and negative predictive value (NPV), except for Ki67, whose analysis involved a comparison of bias between the methods and correlation between lymphocyte subtypes and tumor characteristics. Results: The comparison between FC and IHC for each marker presented the ER analysis (sensitivity: 75\%, specificity: 90\%, PPV: 96.7\%, NPV: 47.4\%); PR analysis (sensitivity: 72\%, specificity: 70\%, PPV: 79.3\%, NPV: 60.8\%); HER2 analysis (sensitivity: $80 \%$, specificity: $90.2 \%$, PPV: $66.7 \%$, NPV: $94.9 \%$ ). The FC Ki67 analysis proved to be equivalent to that of IHC, with the advantage of not having observational bias. No correlations were identified between the profile of the population of intratumoral lymphocytes and the molecular subtype or the histological grade of the tumor. Conclusion: The results show the ability of FC in safely and promptly detecting breast cancer markers used in clinical practice. The use of FC, together with morphological analysis and IHC, might overcome the individual limitations of each methodology, efficiently providing reliable and rapid results, which would lead to faster diagnosis and more accurate prognosis, directly benefiting the patients. 